\title{
An Introduction to the Time-of-Flight Technique
}

\author{
Per Håkansson \\ The Ângström Laboratory, \\ Div. of Ion Physics, Box 534, S-751 21 Uppsala, Sweden
}

Received May, 1999

\begin{abstract}
In the last two decades, several new types of ion sources have been introduced in organic mass spectrometry like bombardment with heavy ions, PDMS, and laser light, MALDI. Hand in hand with the new ionization techniques followed also a renaissance for the 50 year old time-of-flight technique for the mass analyses. In this paper the basics of the ToF technique is described in a tutorial non-theoretical way for the beginner together with some practical hints. The electrostatic mirror, the delayed extraction technique as well as some recent technical developments are also included.
\end{abstract}

\section{Introduction}

A mass spectrometer is build up by three major parts: an ion source to create ions of the sample to be investigated, a mass analyzer to determine the mass distribution of the ions from the source and a detector to detect the ions that have been selected by the mass analyzer. A common mass analyzer is a combination of magnetic and electric fields in a so called sector instrument. Other possibilities are to use quadrupoles or ion traps.

The idea to measure the time that ions, with a known energy, need to travel a certain distance and then calculate their mass was first exploited by Hammer in 1911. The use of ToF in mass spectrometry was consolidated by W. E. Stephans [1] in 1946, Later in 1948, A. E. Cameron and D. F. Eggers [2] at Clinton engineer works, Tennessee, introduced an instrument containing the basic building blocks of a modern ToF instrument: an ion source with an acceleration region followed by a field free region and with a stop detector at the end.

However, the resolving power of a ToF instrument is poor compared to a sector instrument and it was not commonly used until Macfarlane [3] et. al. introduced the PDMS technique, plasma desorption mass spectrometry, in 1974. Then the advantages with the ToF technique became clear: no scanning is needed like in a sector instrument, high transmission, high sensitivity, fast, cheep, simple and in principle unlimited mass range.

Today the ToF technique is well established and used in combination with quite different ion sources like in SIMS [4], secondary ion mass spectrometry, MALDI [5], matrix assisted desorption/ionization and ESI [6], electrospray ionization. There are also hybrid instru- ments like the Q-ToF: a quadrupole is used to select a certain mass, the parent ions. By collisions with gas molecules in a small cell the parent ions will break up into fragments or daughter ions. The masses of the daughter ions are then analyzed with a time-of-flight analyzer. This technique for obtaining structure information about molecules is called MS/MS.

A drawback with the ToF technique has been the relatively poor mass resolving power due to the spread in the initial energies of the ions as well as the spatial distribution of them. However, with the use of the delayed extraction technique [7] and electrostatic mirrors [8] the mass resolving power becomes sufficient for most applications today [9]. In this short paper the basic ToF concepts will be described in a popular way together with references for further reading. A recent review article about ToF has been written by Guilhaus [10] and a nice book by Cotter [11].

\section{The straight spectrometer}

Consider a straight spectrometer as in Fig 1. The sample molecules to be studied are deposited on a metallic target backing kept at the acceleration potential $U$ in front of a grounded grid. The target is bombarded with for e.g. fast heavy ions from an accelerator. The start signal could be generated from the accelerator itself or from a start detector that the beam pass just before the target. The start detector contains a thin foil that produces secondary electrons when the fast heavy ion passes. The burst of electrons is amplified with channel plates. The secondary ions from the target are accelerated into the field free region with length $\mathrm{L}$ where they drift with constant velocity until they reach the stop detector. Depending on how the stop detector is 


\section{FAST ION - SIMS - SETUP}

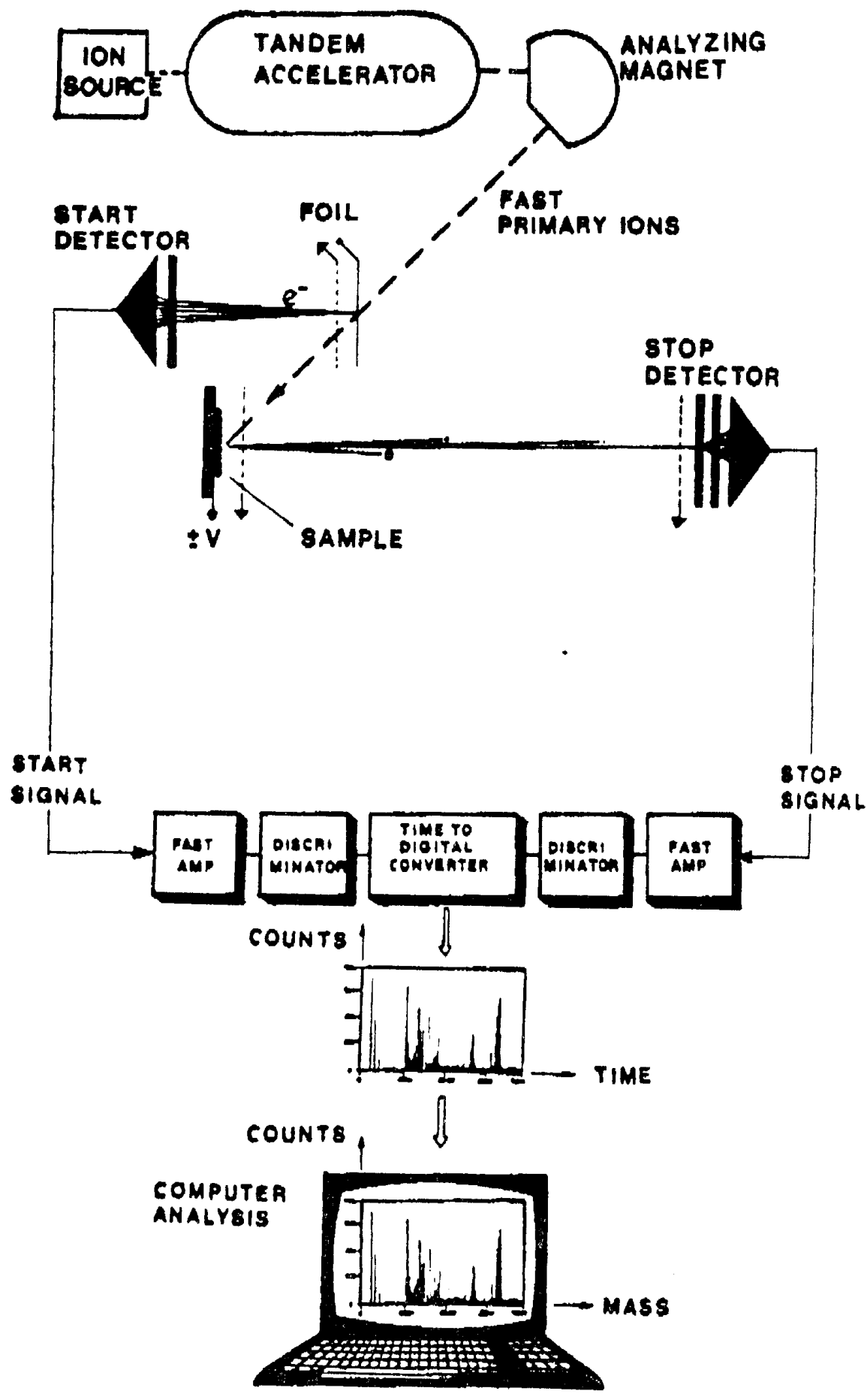

Figure 1. Working principle of a straight time-of-flight mass spectrometer. Molecules are desorbed and ionized when fast heavy ions from an accelerator hit the target at high voltage. The secondary ions are accelerated through a grid and enter a field free drift tube. At the end, the ions are stopped in micro channel plate detector which gives a stop pulse to the digital clock. The start pulse comes from a burst of electrons that are generated when the beam passes a thin foil. The time difference between start and stop pulses are proportional to the square root of the ion mass. The main principle is the same for bombardment with fission fragments from ${ }^{252} \mathrm{Cf}$ source (PDMS) or keV ions (SIMS) or laser light (MALDI). 
The energy of the ions when entering the acceleration region is $q U$. This is equal to kinetic energy $E_{k}=1 / 2 \mathrm{mv}^{2}$, where the velocity $v=L / t$. The basic equation for the time- of- flight is thus

$$
t=L \sqrt{m / q U}
$$

Even if the offset time between the start pulse and the time when the primary ion hits the target and the time spent in the stop detector are taken into account the same simple relation holds:

$$
T o F=A \sqrt{m}+B
$$

where $A$ and $B$ are constants. To mass calibrate an unknown spectrum it is thus enough to determine the ToF for two known masses and then calculate $A$ and $B$.

The start and stop signals are fed into a TDC, time to digital converter. This is a digital clock that will give as output a number that is proportional to the time difference between the start and stop pulses. This number will be the channel address in a spectrum. The content of that channel will increment by 1 . In this way a histogram spectrum will be built up step by step. This way of recording data is called event-by-event mode or single ion counting: all stop signals followed one start signal are registered. To a given start signal, can follow stop signals from light ions as well as from the molecular ion itself. Every event can contain information about the whole mass range. This is in contrast to a magnetic analyzer where the magnetic field is scanned and the intensity of the corresponding masses are recorded.

\section{Detectors}

The standard detector used today is an assembly of two micro channel plates coupled together as in Fig 2. Across the plate is a potential difference of about $1000 \mathrm{~V}$. The plates are built up of small channels that are tilted at an angle with respect to the surface normal. When an ion hits the channel it will produce some electrons that are accelerated into the channel where it will hit the wall and produce more electrons and so on. Each plate has a gain of about 1000. After two plates so many electrons are produced that it is possible to detect a pulse from the anode. The channel plate signals are fast, which is a must for good timing. The rise time of the anode pulse is less than a ns.

The plates can be coupled in two different ways. The anode can be at ground potential, which means that the front end of the first plate must be negatively biased. This has the consequence that positive ions will be postaccelerated but negative ions will be retarded. The anode can also be floated at high positive voltage. In that case, the front plate is at ground potential and the secondary ions will not be influenced in the stop detector region.
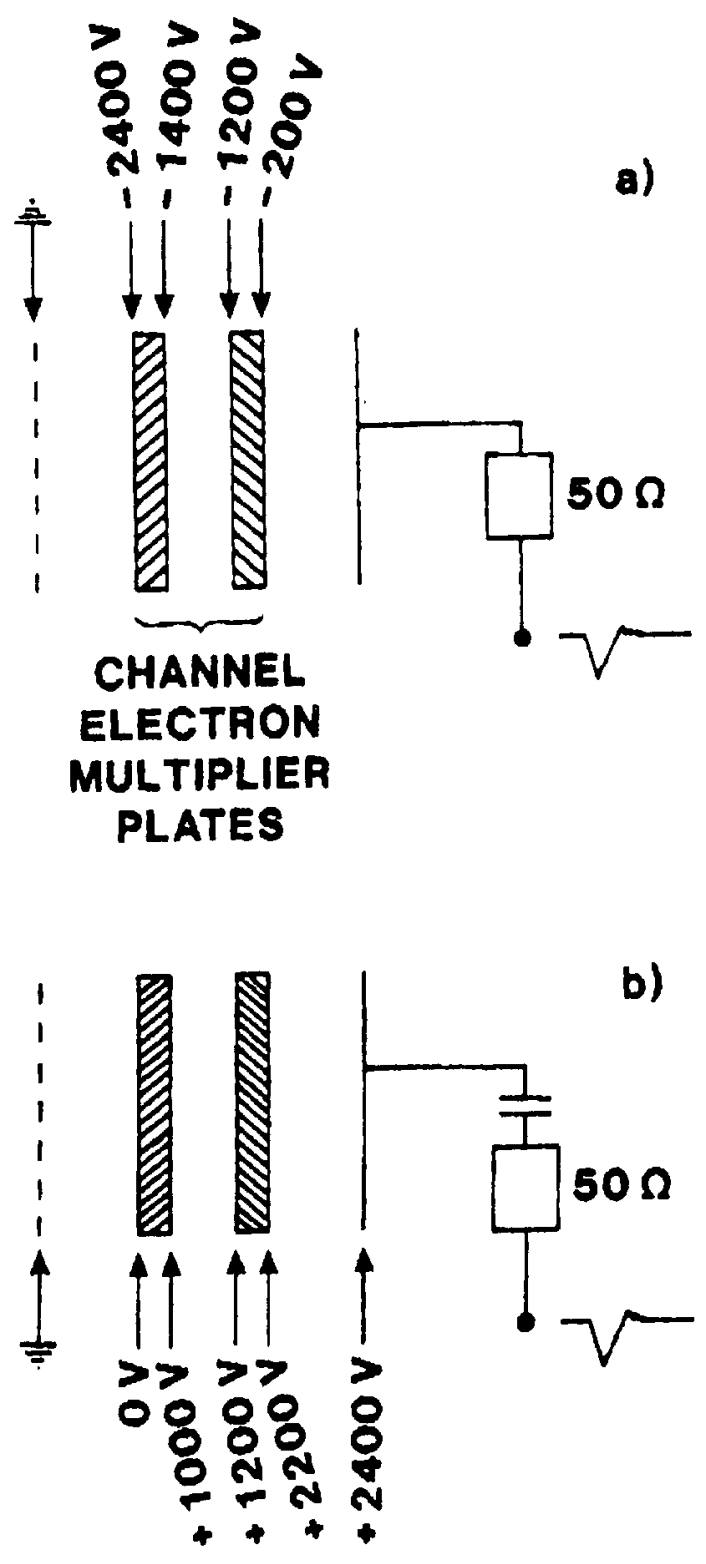

Figure 2. The typical detector in a time-of-flight spectrometer consists of two micro channel plates coupled in tandem. The total gain is about $10^{6}$. The plates can be coupled either with the anode plate grounded (a) or at positive high voltage (b).

To trigger a micro channel plate detector it is in most cases enough to just let the ions hit the front plate directly. However, when the production of secondary electrons is too low, the ion is forced to hit a thin foil in front of the detector. The secondary electrons from the foil will then be accelerated into the plates. To enhance the electron yield and to make it possible to go to high postacceleration voltages, it is also possible to use a converter plate beside the detector. The secondary electrons from the plate are then steered into the detector by a magnetic field.

A drawback of the channel plates is that they have a recovery time in the $\mu$ s time range. This means that if a channel is hit by an ion it will take a certain time 
before it is ready for detecting the next ion. If the stop rate is low like in PDMS or SIMS this effect has no influence, but if many ions come per start like in MALDI the high low-mass count rate will shadow the interesting high-mass ions.

This problem can be resolved in many different ways. One of the channel plates can be pulsed. When the low-mass ions come the detector, it is not active but after a certain time the detector gets full high voltage and can record the high-mass ions. Another way is to use a set of deflection plates or any type of ion gate and deflect away unwanted low-mass ions from the secondary ion beam. The gate is then switched off when the interesting molecules comes.

\section{Electronics}

To record a ToF spectrum it is, in most cases, enough to use a timing amplifier for the detector signal followed by a constant faction discriminator, CFD. This module gives a well-defined timing signal independently of the amplitude of the stop pulse from the detector, the so called "amplitude walk" phenomenon.

In applications with few stops/start the time can be measured with an analog time to pulse height converter, TPHC, or better a digital time converter, TDC. A good converter, like the CTN-M2 built by the IPN at Orsay [12], has a resolution of $0.5 \mathrm{~ns}$, a time range of $256 \mu \mathrm{s}$ and can handle 256 stops per start. The dead time is 20 ns. It sounds more than enough the quantity 256 stops per start, but these stops must be of different masses. If two ions arrive at the same time in the stop-detector the output pulse from the detector will be twice the pulse for one ion, but the TDC will still count it as one ion. In applications with a high probability that many molecular ions with the same mass are desorbed at the same time, like in MALDI, it is therefore better to use a transient recorder or a digital oscilloscope in order not to loose statistics in the peak.

This problem can also be circumvented by using a segmented anode [13] and add up contributions from each part. At IPN the electronic department has also developed a charge recorder that integrates the stop detector peak and give an output pulse proportional to the area.

\section{The electrostatic mirror}

A powerful way of compensating for the spread in the initial energy distribution of the secondary ions is to reflect the beam in an electrostatic field, see Fig 3. Consider a package of ions having the same mass and approaching the mirror after been accelerated to a certain energy. Due to the initial energy distribution some ions will have lower energy (i.e. moving with too low velocity) and some higher energy (i.e. moving too fast) than the correct one. Ions with too high energy will penetrate deeper into the mirror before they are reflected back, compared to the ones with correct energy. Thereby they will loose time. Ions with too low energy will not penetrate so deep into the mirror before they are reflected back, compared to the ones with correct energy. Thereby they will gain time. The net effect is a time focusing of the ion package at the detector plane.

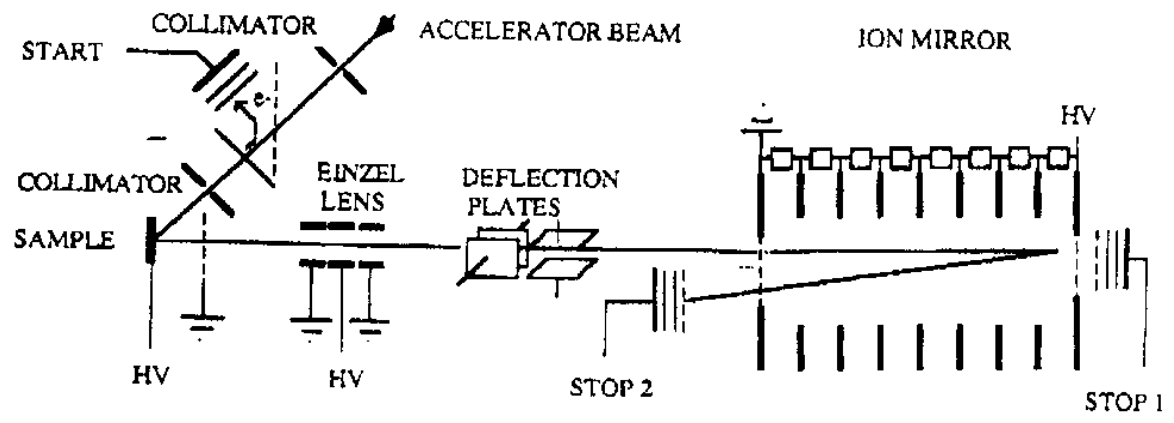

Figure 3. A time-of-flight spectrometer equipped with an electrostatic reflector or ion mirror for improved mass resolving power. The mirror is built up by high precision stainless steel rings separated with high precision ceramic balls. A resistor chain distribute the mirror voltage over the rings uniformly. A stop detector at the rear of the mirror gives the possibility to study metastable decays by coincidence measurements between that detector and the detector for the reflected ions. The spectrometer has also an einzel lens to focus the secondary ion beam and a set of deflection plates for steering the beam correctly into the mirror.

For a single stage mirror, Fig. 3, the length of the mirror should be one quarter of the total field free path which means that the total dimensions of an instrument can be quite large. A more compact mirror is a two stage mirror that compensates for higher order effects in the time-of-flight equation for the system. This is obtained by inserting one more grid in the mirror and set the dimensions and voltages correct. 
The compensating effect on time-of-flight instrument with a mirror can easily be demonstrated by changing the acceleration voltage and monitor the ToF for any ion. With a single stage mirror [14] one can typically change the acceleration voltage $+150 \mathrm{~V}$ and the peak position is the same within $1 \mathrm{~ns}$. For a two stage mirror the compensation is roughly 10 times higher [15].

\section{Delayed extraction}

Delayed extraction or time lag focusing was invented by Wiley \& McLaren already in 1955 . How powerful this technique is became however not clear until Brown [16] and Vestal [17] applied it to MALDI. The working principle is the following. Consider two identical ions that are produced with different initial energies. In the standard ion source geometry with one grid they both gain the same amount of energy in the acceleration process. After the grid, the ion with highest initial energy will continuously increase the distance to the ion coming behind and it will reach the detector first.

Consider now the pulsed case, with an extra grid between the sample and grounded grid, Fig. 4. In the desorption moment, the target and 1st grid are on the same potential. The ion with the highest initial energy will move longer out in the acceleration region than the ion with low initial energy. After a certain delay time, the high voltage is increased on the target to produce a linear acceleration field. However, in this case the slower ion will be accelerated more than the faster ion. By choosing correctly the distances and delay, the ions can be made to reach the stop detector at the same time.

\section{Conclusion}

From being a technique that was hardly accepted 20 years ago, ToF is today well established and the number of commercial instruments on the market increases fast. New concepts and ideas are constantly presented. Vestal et al have developed a ToF MS/MS instrument with a collision cell [18]. Ens et.al. has developed an instrument with a pulsed orthogonal extraction ToF analyzer with two ion sources: electrospray, ESI, or laser light, MALDI [19]. Gamini et al has developed an ion gate for ToF MS/MS experiments with a mass resolving power of up to 5200 [20]. The same group has also demonstrated a mass resolving power of 55000 for molecular ions of substance-P [21] in a MALDI experiment by using multiple reflections in two mirrors facing each other. The era of ToF has just started!

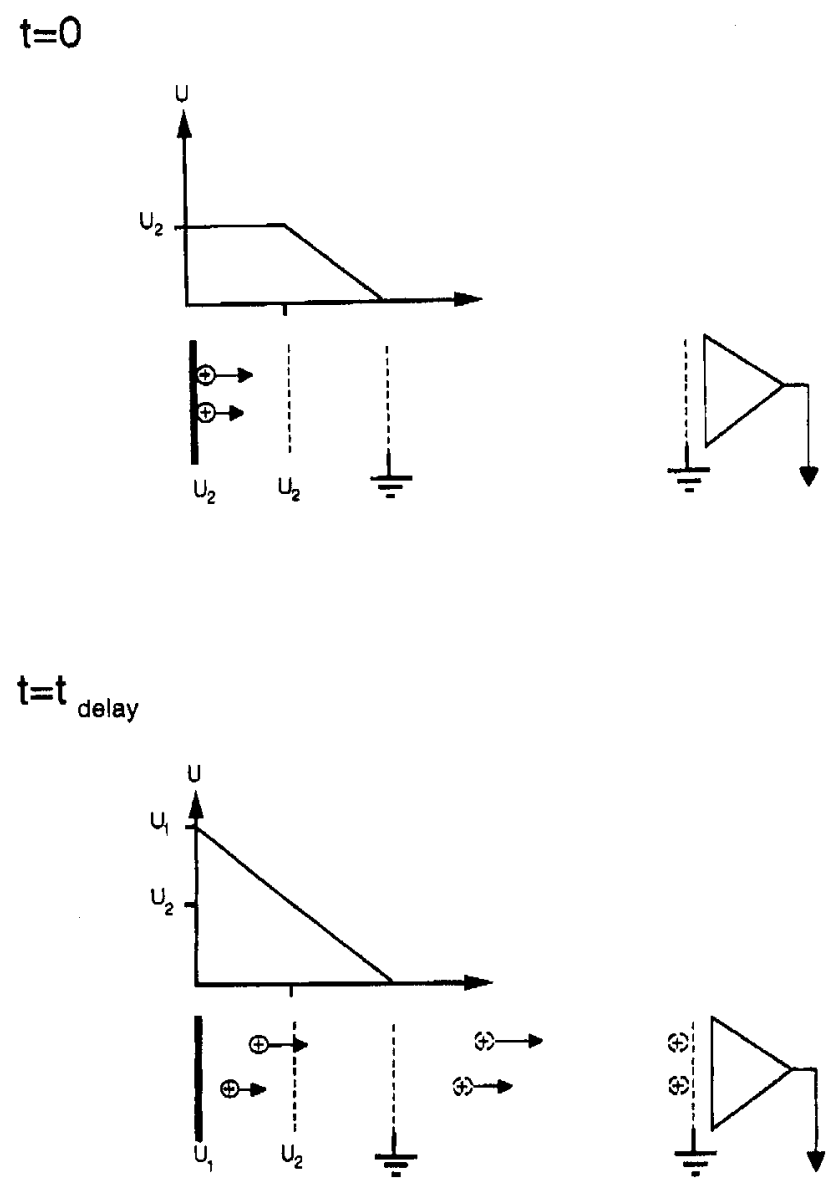

Figure 4. In delayed extraction or time lag focusing an extra grid is mounted between the target and grounded acceleration grid. In the desorption moment, $t=0$, the target and first grid are on the same potential U2. After a certain delay, $t_{\text {delay }}$ the target potential is quickly switched from U2 to $U 1$ and the ions are extracted from the ion source. An ion with high initial energy will be less accelerated than an ion with low initial energy. By choosing the delay time and the distances correctly a net focusing effect can be obtained at the stop detector plane.

\section{References}

[1] W.E. Stephans, Bulletin of the American Physical Society, 21, 22 (1946).

[2] A.E. Cameron and D.F. Eggers, Rev. Sci. Ins., 19, 605 (1948).

[3] D.F. Torgerson, R.P. Skowronski and R.D. Macfarlane Biochem. Biophys. Res. Com. 60, 616 (1974).

[4] W. Ens, K.G. Standing, B.T. Chait and F.H. Field, Anal. Chem. 53, 1241 (1981).

[5] M. Karas, D. Bachmann, U. Bahr and Hillenkamp, Int. J. Mass Spectrom. Ion Process, 78, 53 (1987).

[6] J.G. Boyle, C.M. Whitehouse and J.B. Fenn, Rapid Comm. Mass Spectrom. 5, 400 (1991).

[7] W.C. Wiley and I.H. McLaren, Rev. Sci. Ins., 26, 1150 (1955). 
[8] B.A. Mamyrin, V.J. Karatajev, D.V. Smikk and V.A. Zagulin, Soviet Phys. JEPT 37, 45 (1973).

[9] P. Juhasz, M. L. Vestal and S. A. Martin; Optimization Strategies in Delayed Extraction MALDI TOF, Proceedings of the 45th ASMS Conference on Mass Spectrometry and Allied Topics, Palm Springs, California, June 1-5, 1997, p 1100.

[10] M. Guilhaus, J. Mass Spectrom. 30, 1519 (1995).

[11] R.J. Cotter, Time-of-Flight Mass Spectrometry, ACS Professional Reference Books, American Chemical Society, 1997.

[12] R. Sellem, Service Electronique Physique, Institut de Physique Nucléaire, Bât 102, F-914 06 Orsay, France.

[13] S. Della-Negra et al, The Multi-Impact Localisation Detector LAG 1, Presented at the Desorption '98 conference, Rio de Janeiro, Brazil, September 21-26, 1998.

[14] G. Brinkmalm, P Håkansson, J. Kjellberg, P. Demirev, B. U. R. Sundquist and W. Ens, Int. J. Mass. Spectrom Ion Processes 114, 183 (1992).

[15] A. Brunelle, S. Della-Negra, J. Depauw, H. Joret, Y. Le Beyec, Rapid Commun Mass Spectrom. 5, 40 (1991).
[16] R.S. Brown and J.J. Lennon, Anal. Chem. 67, 1998 (1995).

[17] M.L. Vestal, P. Juhasz and S.A. Martin, Rapid Comm. Mass Spectrom. 9, 1044 (1995).

[18] M. Vestal, P. Juhasz, W, Hines and S. Martin; An Improved Delayed Extraction MALDI TOF MS for PSD and CID Proceedings of the 46th ASMS Conference on Mass Spectrometry and Allied Topics, Orlando, Florida, May 31-June 4, 1998, p 35.

19. W. Ens et al, A MALDI Source with Collisional Cooling for Orthogonal-Injection TOF Presented at the Desorption ' 98 conference, Rio de Janeiro, Brazil, September 21-26, 1998.

[19] C. K. G. Piyadasa, P. Håkansson, T. R. Ariyaratne and D. F. Barofsky Rapid. Commun. Mass. Spectrom. 12, 1655 (1998).

[20] C. K. G. Piyadasa, P. Håkansson, T. R. Ariyaratne, Submitted to Rapid. Commun. Mass. Spectrom. 